\title{
A NUMERICAL SOLUTION OF THE ADVECTION-DIFFUSION EQUATION BY USING EXTENDED CUBIC B-SPLINE FUNCTIONS
}

\author{
Melis ZORŞAHIN GÖRGÜLÜ ${ }^{1, *}$, İdris DAĞ ${ }^{2}$, Sümeyye DOĞAN ${ }^{1}$, Dursun IRK ${ }^{1}$ \\ ${ }^{1}$ Mathematisc-Computer Department, Science and Art Faculty, Eskişehir Osmangazi University, Eskişehir, Turkey \\ ${ }^{2}$ Computer Engineering Department, Science and Art Faculty, Eskişehir Osmangazi University, Eskişehir, Turkey
}

\begin{abstract}
In this paper, numerical solution of the advection-diffusion equation is obtained by using extended cubic B-spline functions. For space discretization, the extended cubic B-spline Galerkin method is used to integrate the advection-diffusion equation and for time discretization, the Crank-Nicolson method is employed to obtain the fully integrated advection-diffusion equation. The maximum error norm has been used to show the accuracy of the method. Robustness of the suggested method is shown by studying some classical test problems and comparing the results with some earlier ones.
\end{abstract}

Keywords: Extended cubic B-spline, Galerkin finite element method, Advection-diffusion equation

\section{INTRODUCTION}

It is well-known that many real life problems in physics and engineering can be modelled by the advection-diffusion equation (ADE) which describes phenomena including weak nonlinearity and dispersion waves. Since the solutions of the ADE include the sharp behavior with some selection of parameters, the numerical methods are of interest due to modelling of steep solutions. Many studies have been existed for the numerical solutions of the ADE using spline functions so far [1-23]. By keeping the continuity, adding higher order terms with a free parameter to the B-spline which is a piecewise function, the extended B-spline function is obtained. The use of different free parameters changes the shape of extended B-spline. The effect of the additional term and free parameters for the extended B-splines are discussed in the studies [24, 25].

Our aim in this work is to investigate the results obtained by using extended B-spline functions with a combination of Galerkin and Crank-Nicolson methods to find numerical solutions of the ADE. Although the extended B-spline function has not yet been extensively used for the numerical solutions of partial differential equations, several studies are available in the literature [14, 26, 27]. In parallel with those, we use the extended cubic B-spline Galerkin method (ECBSGM) that is not performed before.

\section{GOVERNING EQUATION}

The one dimensional ADE which describes the transport and diffusion processes is

$$
\frac{\partial u}{\partial t}+\xi \frac{\partial u}{\partial x}-\mu \frac{\partial^{2} u}{\partial x^{2}}=0,
$$

where the function $u(x, t)$ indicates the concentration at position $x$ and time $t, \xi$ and $\mu$ represent the uniform flow velocity parameter and the constant diffusion coefficient, respectively. The initial and boundary conditions of Eq. (1) are

*Corresponding Author: mzorsahin@ogu.edu.tr

Receiving Date: 25 August 2017 Publishing Date: 29 June 2018 


$$
\begin{gathered}
u(x, 0)=u_{0}(x), \quad 0 \leq x \leq L \\
u(0, t)=f_{0}(t), \quad u(L, t)=f_{L}(t) \quad \text { or }-\left.\mu \frac{\partial u}{\partial x}\right|_{L}=\varphi_{L}(t)
\end{gathered}
$$

where the parameters $L$ and $\varphi_{L}$ demonstrate the length of the channel and the flux at the boundary $x=L$, respectively and $u_{0}, f_{0}, f_{L}$ are imposed functions.

\section{EXTENDED CUBIC B-SPLINE GALERKIN METHOD}

Let we take a uniform mesh with the knots $x_{l}=x_{0}+l h$ on $[a, b]$ and $Q_{l}(x)$ be the extended cubic Bsplines at these points with knots $x_{l}, l=-3,-2,-1, N+1, N+2, N+3$ outside the interval $[a, b]$ and having a finite support on the four consecutive intervals $\left[x_{l}+k h, x_{l}+(k+1) h\right]_{k=-3}^{0}, l=0, \ldots, N+2$. The extended cubic B-spline is given as

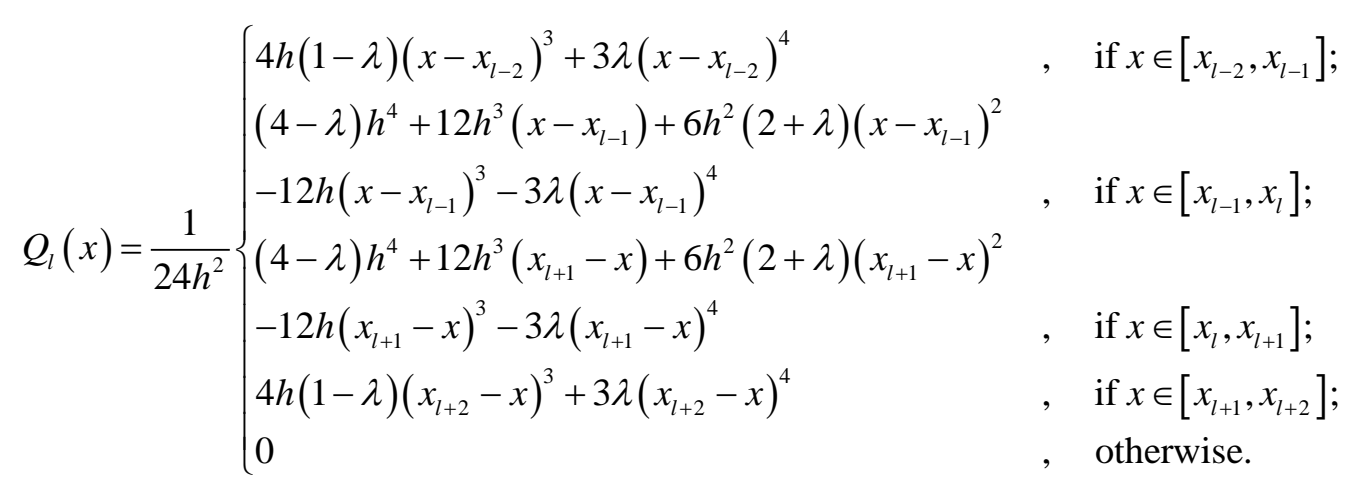

The values of $Q_{l}(x), Q_{l}^{\prime}(x)$ and $Q_{l}^{\prime \prime}(x)$ at the knots $x_{l}$ 's are tabulated in Table 1. The $Q_{l}(x)$, $l=-1, \mathrm{~K}, N+1$ form a basis for functions defined on the interval $[a, b]$.

Table 1. Extended cubic B-spline values at the knot points

\begin{tabular}{cccccc}
\hline & $x_{l-2}$ & $x_{l-1}$ & $x_{l}$ & $x_{l+1}$ & $x_{l+2}$ \\
\hline$Q_{l}(x)$ & 0 & $\frac{4-\lambda}{24}$ & $\frac{8+\lambda}{12}$ & $\frac{4-\lambda}{24}$ & 0 \\
$Q_{l}^{\prime}(x)$ & 0 & $\frac{1}{2 h}$ & 0 & $-\frac{1}{2 h}$ & 0 \\
$Q_{l}^{\prime \prime}(x)$ & 0 & $\frac{2+\lambda}{2 h^{2}}$ & $-\frac{2+\lambda}{h^{2}}$ & $\frac{2+\lambda}{2 h^{2}}$ & 0 \\
\hline
\end{tabular}

We seek an approximation $U$ to the analytical solution $u$ in terms for the extended cubic B-splines

$$
u(x, t) \approx U(x, t)=\sum_{l=-1}^{N+1} Q_{l}(x) \delta_{l}(t)
$$

where $\delta_{l}(t)$ are unknown parameters to be obtained by Galerkin approach to the ADE. $U$ and its derivatives can be calculated at each knot by the following equations: 


$$
\begin{aligned}
& U_{l}=U\left(x_{l}, t\right)=\frac{4-\lambda}{24} \delta_{l-1}+\frac{8+\lambda}{12} \delta_{l}+\frac{4-\lambda}{24} \delta_{l+1}, \\
& U_{l}^{\prime}=U^{\prime}\left(x_{l}, t\right)=-\frac{1}{2 h} \delta_{l-1}+\frac{1}{2 h} \delta_{l+1}, \\
& U_{l}^{\prime \prime}=U^{\prime \prime}\left(x_{l}, t\right)=\frac{2+\lambda}{2 h^{2}} \delta_{l-1}-\frac{2+\lambda}{h^{2}} \delta_{l}+\frac{2+\lambda}{2 h^{2}} \delta_{l+1} .
\end{aligned}
$$

When we choose the extended cubic B-splines as weight functions over the interval $[0, L]$, we obtain following integral for the ADE by Galerkin method:

$$
\int_{0}^{L} Q_{l}(x)\left(u_{t}+\xi u_{x}-\mu u_{x x}\right) d x=0
$$

Over the sub element $\left[x_{k}, x_{k+1}\right]$, Eqs. (5) and (7) can be rewritten as

$$
U^{e}=Q_{k-1}(x) \delta_{k-1}(t)+Q_{k}(x) \delta_{k}(t)+Q_{k+1}(x) \delta_{k+1}(t)+Q_{k+2}(x) \delta_{k+2}(t)
$$

and

$$
\int_{x_{k}}^{x_{k+1}} Q_{j}(x)\left(u_{t}+\xi u_{x}-\mu u_{x x}\right) d x
$$

respectively, where quantities $\delta_{i}(t), i=k-1, \ldots, k+2$ are element parameters and $Q_{j}(x)$, $j=k-1, \ldots, k+2$ are known as the element shape functions.

According to the Galerkin discretization scheme, replacing $U_{t}, U_{x}, U_{x x}$ which are derivatives of the approximate solution $U^{e}$ in Eq. (8), into $u_{t}, u_{x}, u_{x x}$ which are derivatives of the exact solution $u$, respectively, we have

$$
\sum_{i=k-1}^{k+2}\left\{\left(\int_{x_{k}}^{x_{k+1}} Q_{j} Q_{i} d x\right) \dot{\delta}_{i}+\xi\left(\int_{x_{k}}^{x_{k+1}} Q_{j} Q_{i}^{\prime} d x\right) \delta_{i}-\mu\left(\int_{x_{k}}^{x_{k+1}} Q_{j} Q_{i}^{\prime \prime} d x\right) \delta_{i}\right\}
$$

where $j=k-1, \ldots, k+2 ; k=0,1, \mathrm{~K}, N-1$ and ${ }^{\bullet}$ symbolizes time derivative.

Let the integrals in Eq. (10) are denoted by

$$
P_{j i}^{e}=\int_{x_{k}}^{x_{k+1}} Q_{j} Q_{i} d x, \quad R_{j i}^{e}=\int_{x_{k}}^{x_{k+1}} Q_{j} Q_{i}^{\prime} d x, \quad S_{j i}^{e}=\int_{x_{k}}^{x_{k+1}} Q_{j} Q_{i}^{\prime \prime} d x
$$

and $\mathbf{P}^{e}, \mathbf{R}^{e}$ and $\mathbf{S}^{e}$ are the element matrices of which dimensions are $4 \times 4$. With these matrices we can write the following instead of the (10):

$$
\mathbf{P}^{e} \dot{\boldsymbol{\delta}}^{e}+\left(\xi \mathbf{R}^{e}-\mu \mathbf{S}^{e}\right) \boldsymbol{\delta}^{e}
$$


where $\boldsymbol{\delta}^{e}=\left(\delta_{k-1}, \ldots, \delta_{k+2}\right)^{T}$.

Collecting the systems (12) over all elements, the following global system can be written

$$
\mathbf{P} \dot{\boldsymbol{\delta}}+(\xi \mathbf{R}-\mu \mathbf{S}) \boldsymbol{\delta}=0
$$

where $\mathbf{P}, \mathbf{R}$ and $\mathbf{S}$ are reproduced by the corresponding element matrices $\mathbf{P}^{e}, \mathbf{R}^{e}$ and $\mathbf{S}^{e}$ and $\boldsymbol{\delta}=\left(\delta_{-1}, \ldots, \delta_{N+1}\right)^{T}$ includes all element parameters

By using the Crank-Nicolson method for the unknown parameters $\delta$

$$
\boldsymbol{\delta}=\frac{\delta^{n+1}+\delta^{n}}{2}, \quad \dot{\delta}=\frac{\delta^{n+1}-\delta^{n}}{\Delta t}
$$

we have the following iterative formula:

$$
\left[\mathbf{P}+\frac{\Delta t}{2}(\xi \mathbf{R}-\mu \mathbf{S})\right] \boldsymbol{\delta}^{n+1}=\left[\mathbf{P}-\frac{\Delta t}{2}(\xi \mathbf{R}-\mu \mathbf{S})\right] \boldsymbol{\delta}^{n}
$$

To incorporate boundary conditions into the remaining system (14) before starting the iteration process, we eliminate the terms $\delta_{-1}^{n+1}$ and $\delta_{N+1}^{n+1}$ from the system with the following equations:

$$
\begin{aligned}
& u(0, t)=\frac{4-\lambda}{24} \delta_{-1}^{n}+\frac{8+\lambda}{12} \delta_{0}^{n}+\frac{4-\lambda}{24} \delta_{1}^{n}=f_{0}(t), \\
& u(L, t)=\frac{4-\lambda}{24} \delta_{N-1}^{n}+\frac{8+\lambda}{12} \delta_{N}^{n}+\frac{4-\lambda}{24} \delta_{N+1}^{n}=f_{L}(t) .
\end{aligned}
$$

Thus, a septa-diagonal matrix is obtained.

To carry on the iteration of the system (14), the initial parameters $\delta^{0}$ must be obtained from the initial condition (2) and the derivatives of the boundary conditions (3) at both ends:

$$
\begin{aligned}
& u^{\prime}\left(x_{0}, 0\right)=-\frac{1}{2 h} \delta_{-1}+\frac{1}{2 h} \delta_{1} \\
& u\left(x_{k}, 0\right)=\frac{4-\lambda}{24} \delta_{k-1}+\frac{8+\lambda}{12} \delta_{k}+\frac{4-\lambda}{24} \delta_{k+1}, \quad k=0, \ldots, N, \\
& u^{\prime}\left(x_{N}, 0\right)=-\frac{1}{2 h} \delta_{N-1}+\frac{1}{2 h} \delta_{N+1} .
\end{aligned}
$$

The matrix equation (15) is solved by the way of Thomas algorithm. Thus the approximate solution $U$ (5) can be determined by using these $\delta$ values.

\section{TEST PROBLEMS}

In this section, two test problems are addressed where the correctness of given algorithm is measured by error norm 


$$
L_{\infty}=\left\|u^{\text {exact }}-u^{\text {numeric }}\right\|_{\infty}=\max _{0 \leq j \leq N}\left|u_{j}^{\text {exact }}-u_{j}^{\text {numeric }}\right| \text {. }
$$

In numerical calculations, the value of $\lambda$ in the extended cubic B-spline is experimentally selected by scanning the predetermined interval with a small increment in a way that the best numerical solutions are obtained for the test problems. The Courant number is defined as

$$
C_{r}=\xi \frac{\Delta t}{h}
$$

\subsection{First Problem}

In the first example, we consider the pure advection that is $\mu=0$, in an infinitely long channel is of long constant cross-section, bottom slope and in which constant velocity is $\xi=0.5 \mathrm{~m} / \mathrm{s}$. The analytical solution is

$$
u(x, t)=10 \exp \left(-\frac{1}{2 \rho^{2}}\left(x-x_{0}-\xi t\right)^{2}\right)
$$

where the standard deviation is chosen as $\rho=264 \mathrm{~m}$ and the distribution is $x_{0}=2 \mathrm{~km}$ away from the start at the beginning. The initial concentration can be obtained from (17) by taking $t=0$. At the boundaries of interval [0,9000], the values of Eq. (17) are chosen as zero. Figure 1 shows the transportation of initial distribution after $t=9600 \mathrm{~s}$.

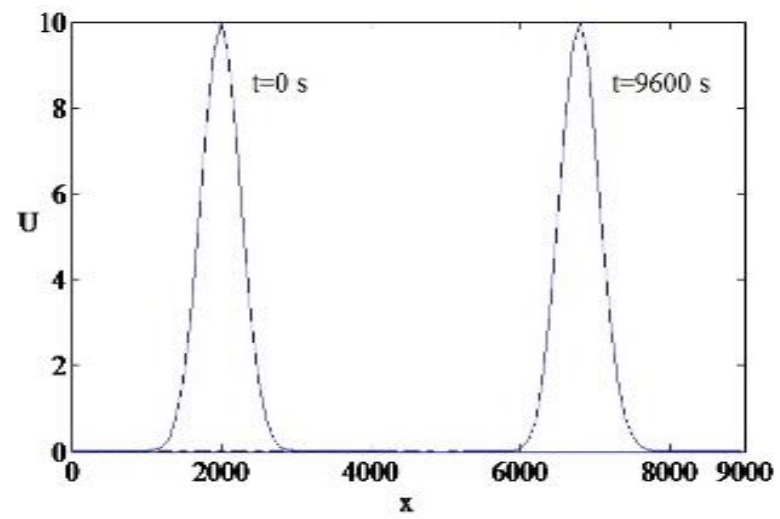

Figure 1. Transportation of the initial distribution with $C_{r}=0.25$ and $\Delta t=50$.

To see the errors along the whole domain for various Courant numbers, Table 2 is documented. According to this table, the results of ECBSGM for various Courant numbers are obtained to be same accuracy with those produced by Dag et al. [5] and Irk et al. [14]. For various values of $\lambda$, Table 3 is shown the errors with $C_{r}=0.25$ at $t=9600 \mathrm{~s}$.

Table 2. Errors at $t=9600 \mathrm{~s}$ with $\xi=0.5 \mathrm{~m} / \mathrm{s}$.

\begin{tabular}{ccccccc}
\hline$C_{r}$ & $h$ & $\Delta t$ & $\lambda$ & ECBSGM & {$[14]$} & {$[5]$} \\
\hline 0.125 & 200 & 50 & -0.568169 & $2.18 \mathrm{E}-1$ & 1.29 & $5.18 \mathrm{E}-1$ \\
0.25 & 100 & 50 & -0.142055 & $1.90 \mathrm{E}-1$ & $3.25 \mathrm{E}-1$ & $3.76 \mathrm{E}-1$ \\
0.50 & 50 & 50 & -0.032925 & $1.90 \mathrm{E}-1$ & $1.98 \mathrm{E}-1$ & $3.73 \mathrm{E}-1$ \\
0.50 & 10 & 10 & -0.027064 & $7.50 \mathrm{E}-3$ & $7.51 \mathrm{E}-3$ & \\
0.50 & 1 & 1 & -0.0 .27064 & $7.50 \mathrm{E}-5$ & $7.50 \mathrm{E}-5$ & \\
0.50 & 0.5 & 0.5 & -0.498015 & $1.88 \mathrm{E}-5$ & $1.88 \mathrm{E}-5$ & \\
\hline
\end{tabular}


Table 3. Errors at $t=9600 \mathrm{~s}$ with $\xi=0.5 \mathrm{~m} / \mathrm{s}$ and $C_{r}=0.25$.

\begin{tabular}{cc}
\hline$\lambda$ & ECBSGM \\
\hline-10 & 0.9200226 \\
-5 & 0.4351456 \\
-1 & 0.1968777 \\
-0.5 & 0.1908966 \\
-0.142055 & 0.1896909 \\
0 & 0.1898749 \\
0.142055 & 0.1904208 \\
0.5 & 0.1933445 \\
1 & 0.2009420 \\
5 & 0.3666941 \\
10 & 0.6572077 \\
\hline
\end{tabular}

The absolute error distribution of the ECBSGM at $t=9600$ is illustrated in Figure 2. Maximum error occurs around the peak concentration.

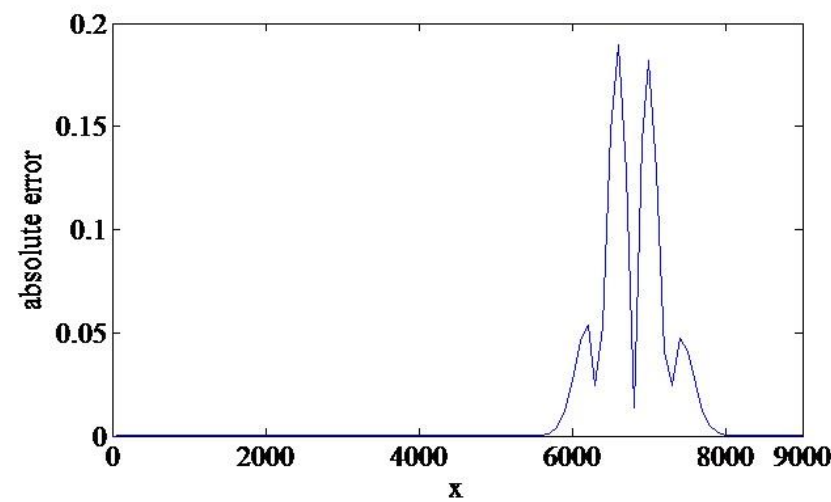

Figure 2. Absolute error distribution at $t=9600 \mathrm{~s}$ with $C_{r}=0.25$ and $\Delta t=50$.

\subsection{Second Problem}

As a second test problem, we deal with both advection and diffusion. The analytical solution to the onedimensional ADE of a Gaussian pulse of unit height over the domain $[0,9]$ is given as

$$
u(x, t)=\frac{1}{\sqrt{4 t+1}} \exp \left(-\frac{\left(x-x_{0}-\xi t\right)^{2}}{\mu \sqrt{4 t+1}}\right)
$$

where $\xi$ is the velocity, $\mu$ is diffusion coefficient and $x_{0}$ is the center of the initial Gaussian pulse [28].

The initial condition is chosen as the analytical value of the Eq. (18) for $t=0$ and the boundary conditions are chosen as

$$
u(0, t)=u(9, t)=0 .
$$

The results presented here are computed for time step $\Delta t=0.0125 \mathrm{~s}$. Parameters in the equation are used as $\mu=0.005 \mathrm{~m}^{2} / \mathrm{s}$ and $\xi=0.8 \mathrm{~m} / \mathrm{s}$. Figure 3 shows the behavior of the numerical solutions for 
various times until the simulation terminating time $t=5$. Thus, the decay in time of the initial pulse is modeled. So that the effect of the diffusion term has been observed in this test problem. The absolute error distribution of the ECBSGM at $t=5$ is illustrated in Figure 4.

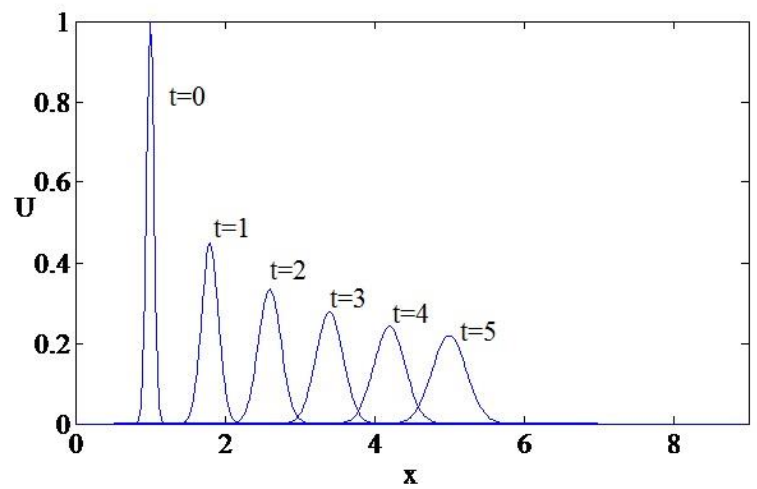

Figure 3. Distributions of an initial Gaussian pulse

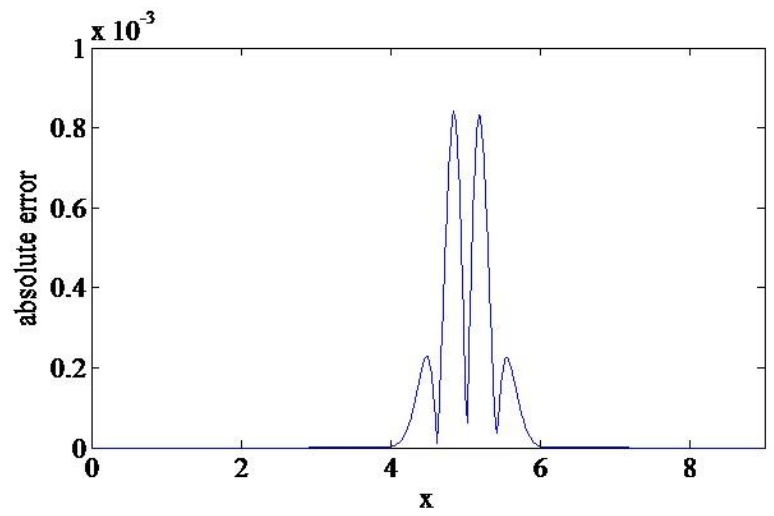

Figure 4. Absolute error distribution at $t=5$ with $h=0.025, \Delta t=0.0125$.

For comparison, the ADE is solved for various Courant numbers and computed errors at $t=5 \mathrm{~s}$ are presented in Table 4.

Table 4. Error norm at $t=5, \xi=0.8 \mathrm{~m} / \mathrm{s}, \mu=0.005 \mathrm{~m}^{2} / \mathrm{s}, \Delta t=0.0125$.

\begin{tabular}{cccccc}
\hline$C_{r}$ & $h$ & $\lambda$ & ECBSGM & Method I [16] & Method II [16] \\
\hline 0.05 & 0.2 & -0.238247 & 0.1326156 & 0.1253926 & 0.1361437 \\
0.10 & 0.1 & -0.238247 & 0.0042296 & 0.0069553 & 0.0145554 \\
0.20 & 0.05 & -0.200000 & 0.0008429 & 0.0012117 & 0.0002886 \\
0.40 & 0.025 & -0.106431 & 0.0008426 & 0.0003071 & 0.0000181 \\
\hline
\end{tabular}

\section{CONCLUSION}

In this paper, we have proposed a new algorithm for the numerical solution of the ADE. This algorithm is obtained by employing extended cubic B-spline functions to the well-known Galerkin finite element method. To see achievement of the method, two test problems is studied. The resulting numerical solutions for various Courant numbers are compared with the previous studies in Tables 2 and 4 . Accordingly, we can say that the proposed method gives acceptable results. 


\section{ACKNOWLEDGEMENTS}

This paper was presented at International Conference on Mathematics and Mathematics Education (ICMME-2017) in Sanliurfa, Turkey.

\section{REFERENCES}

[1] Ahmad Z. Numerical solution for advection-diffusion equation with spatially variable coefficients. ISH Journal of Hydraulic Engineering, 2000; 1: 46-54.

[2] Ahmad Z, Kothyari UC. Time-line cubic spline interpolation scheme for solution of advection equation. Comput Fluids, 2001; 30: 737-752.

[3] Boztosun I, Charafi A, Zerroukat M, Djidjeli K. Thin-plate spline radial basis function scheme for advection-diffusion problems. Electronic Journal of Boundary Elements, 2002; 2: 267-282.

[4] Bulut H, Akturk T, Ucar Y. The solution of advection diffusion equation by the finite elements method. International Journal of Basic\&Applied Sciences, IJBAS-IJENS 2013, 13: 267-282.

[5] Dag I, Irk D, Tombul M. Least-squares finite element method for advection-diffusion equation. Appl Math Comput, 2006; 173: 554-565.

[6] Dag I, Canivar A, Sahin A. Taylor-Galerkin method for advection-diffusion equation. Kybernetes 2011; 40: 762-777.

[7] Dhawan S, Rawat S, Kumar S, Kapoor S. Solution of advection diffusion equation using finite element method. In: Modelling simulation and applied optimization international conference (ICMSAO); 19-21 April 2011; Kuala Lumpur, Malaysia. IEEE. pp. 1-4.

[8] Dhawan S, Kapoor S, Kumar S. Numerical method for advection-diffusion equation using FEM and B-splines. Journal of Computational Science 2012; 3: 429-443.

[9] Funaro D, Pontrelli G. Spline approximation of advection-diffusion problems using upwind type collocation nodes. J Comput Appl Math 1999; 10: 141-153.

[10] Gardner LRT, Dag I. A numerical solution of the advection-diffusion equation using B-spline finite element. In: International AMSE Conference, Systems Analysis, Control \& Design; 4-6 July 1994; Lyon, France. pp. 109-116.

[11] Gardner LRT, Gardner GAS, Netter E. A new B-spline finite element method for the advectiondiffusion equation. In: International Colloquium on Differential equations; 18-23 August 1996; Plovdiv, Bulgaria. pp. 123-152.

[12] Goh J, Majid AA, Ismail AIMd. A comparison of some splines-based methods for the onedimensional heat equation. World Academy of Science, Engineering and Technology, 2010; 70: 858-861.

[13] Goh J, Majid AA, Ismail AIMd. Ismail, Cubic B-spline collocation method for one-dimensional heat and advection-diffusion equations. Journal of Applied Mathematics, 2012; 2012: 1-8. 
[14] Irk D, Dag I, Tombul M. Extended cubic B-spline solution of the advection-diffusion equation. KSCE Journal of Civil Engineers, 2015; 19: 929-934.

[15] Kapoor S, Dhawan S. B-spline finite element technique for advection-diffusion equation. International Journal of Applied Mathematics and Mechanics, 2010; 6: 75-94.

[16] Korkmaz A, Dag I. Cubic B-spline differential quadrature methods for the advection-diffusion equation. Int J Numer Method H, 2012; 22: 1021-1036.

[17] Okamoto S, Sakai K, Matsumoto K, Horiuchi K, Kobayashi K. Development and application of a three-dimensional Taylor-Galerkin numerical model for air quality simulation near roadway tunnel portals. J Appl Meteor, 1998; 37: 1010-1025.

[18] Pepper DW, Kern CD, Long PEJr. Modeling the dispersion of atmospheric pollution using cubic splines and chapeau functions. Atmos Environ, 1979; 13: 223-237.

[19] Szymkiewicz R. Solution of the advection-diffusion equation using the spline function and finite elements. Commun Numer Meth En, 1993; 9: 197-206.

[20] Tsai TL, Chiang SW, Yang JC, Examination of characteristics method with cubic interpolation for advection-diffusion equation. Comput Fluids, 2006, 35: 1217-1227.

[21] Thongmoon M, Mckibbin R. A comparison of some numerical methods for the advection-diffusion equation. Res Lett Int Math Sci, 2006; 10: 49-62.

[22] Thongmoon M, Thangmanee S, Mckibbin R. A comparison of splines interpolation with standard finite methods for one-dimensional advection-diffusion equation. Int J Mod Phys C, 2008; 9: 12911304.

[23] Zoppou C, Roberts S, Renka RJ. Exponential spline interpolation in characteristic based scheme for solving the advection-diffusion equation. Int J Numer Meth Fl, 2000; 33: 429-452.

[24] Hamid NNA, Majid AA, Ismail AIMd. Extended cubic B-spline interpolation method applied to linear two-point boundary value problems. World Academy of Science, Engineering and Technology, 2010; 38: 566-568.

[25] Hamid NNA, Majid AA, Ismail AIMd. Extended cubic B-spline method for linear two-point boundary value problems. Sains Malaysiana 2011; 40: 1285-1290.

[26] Dag I, Irk D, Sari M. The extended cubic B-spline algorithm for a modified regularized long wave equation. Chinese Phys B, 2013; 22: 1-6.

[27] Goh J, Majid AA, Ismail AIMd. Extended cubic uniform B-spline for a class of singular boundary value problems. Science Asia, 2011; 37: 79-82.

[28] Sankaranarayanan S, Shankar NJ, Cheong HF. Three-dimensional finite difference model for transport of conservative pollutants. Ocean Eng, 1998; 25: 425-442. 